\title{
Causes and Prevalence of Tuberculosis from Sputum of Patients based on Sd Bioline $\circledR$ and Genotype Mbtc $®$ Assay in Gombe State, Nigeria
}

Ibrahim $\mathbf{S}^{1^{*}}$, Abubakar UB ${ }^{1}$, Danbirni $\mathbf{S}^{1}$; Usman $\mathbf{A}^{1}$, Kudi AC ${ }^{1}$, Loveth $\mathrm{L}^{2}$, Abdulrazak $\mathrm{H}^{3}$ and Abdulkadir IA ${ }^{1}$

${ }^{1}$ Department of Veterinary Medicine, Ahmadu Bello University, Nigeria

${ }^{2}$ Department of Public Health and Preventive Medicine, Bingham University, Nigeria

${ }^{3}$ Aminu Kano Teaching Hospital, Bayero University, Nigeria

\section{Research Article}

Volume 2 Issue 1

Received Date: February 02, 2017

Published Date: March 10, 2017

DOI: $10.23880 /$ oajmb-16000114

*Corresponding author: Ibrahim S, Department of Veterinary Medicine, Faculty of Veterinary Medicine, Ahmadu Bello University, Zaria, Nigeria, E-mail: sihadejia@gmail.com

\section{Abstract}

This study was carried out in Gombe State, Nigeria to estimate the prevalence and causes of tuberculosis in patients that were suspected to have clinical symptoms compatible to tuberculosis. 1250 patients were included in the study obtained from the medical records of the patients. Five (5) general hospitals were selected for the study. Three sputum samples were collected from the patients and their HIV statuses were also determined using the WHO standard recommended kits called Determine, Unigold and Star pack. Also, data pertaining each individual such as age, sex, occupation and smoking habit were obtained and recorded. The sputum samples were cultured on Loewenstein-Jensen media supplemented with either glycerol or pyruvate. All colonies were ZN stained and the acid fast bacilli (AFB) positive were subjected to SD Bioline ${ }^{\circledR}$ TB AgMPT64 analysis, which differentiate them into the Mycobacterium tuberculosis complex (MBTC) and Non -tuberculosis mycobacteria (NTM). The MBTC were then tested for genetic identification into their various species by the use of Genotype MBTC (Hainlifescience) assay. The results showed that, out of 100 sputum samples collected, 40(40\%) were MBTC and the remaining 60(60\%) were NTM. Of the 40 MBTC subjected to Genotype MBTC, 30(75\%) were Mycobacterium tuberculosis, 6(15\%) were Mycobacterium bovis and 3(7.5\%) were Mycobacterium africanum, $1(2.5 \%)$ was unidentified using the standard banding patterns. Also, one thousand two hundred and fifty(1250) patients records were analyzed, which shows that, age group between 11-40yrs and males had the highest prevalence of $\mathrm{Tb}$ infection with 687(54.96\%) than females with 563(45.04\%), there was a statistical association between sex and also between the age group $(\mathrm{p}<0.05)$. Three hundred and twenty two (322) $(25.76 \%)$ patients tested positive for 


\section{Open Access Journal of Microbiology \& Biotechnology}

HIV with 115(35.71\%) having TB and HIV co-infections. The study therefore had shown that there TB and HIV are present in the State, as such further studies are needed to isolate and differentiate the MBTC and the NTM using clinical specimens in the study area.

Keywords: Sd Bioline®; Genotype Mbtc®; Sputum; Tuberculosis

\section{Introduction}

Members of the genus Mycobacterium tuberculosis complex comprises of M. tuberculosis, M. bovis, M. canetti, M. microti and M. africanum are responsible for the chronic, contagious and highly infectious disease of animals and humans called tuberculosis. Mycobacterium tuberculosis is often isolated from clinical specimen followed by M. bovis and perhaps other members of the complex. In Nigeria, very little is known on the epidemiology of MBTC species associated with tuberculosis. Knowledge on the circulating MBTC species between humans to human or human to animals and vice versa is essential to guide clinicians and policy makers in diagnosis and therapeutic measures and also measures to take in the control of this major public health menace in the country especially with the prevailing increase of $\mathrm{Tb}$ cases due to HIV epidemic. It has been reported by National Agency for the control of AIDs (NACA) that, over 3 million people live with HIV/AIDs in Nigeria with a National prevalence of the disease estimated at $4.1 \%$ in 2010.

The members of the MBTC species caused tuberculosis which is clinically similar and every difficult to distinguish, making surveillance and tracking of species related to an epidemic a very difficult task. Tuberculosis caused by $M$. bovis is similar to that caused by $M$. $t b$ in clinical, pathological and radiological presentations, similarly, it has similarities with M. africanum. Therefore, in case of identification, mistakes are likely to occur where M. bovis and M. africanum are known to co-exist. Newer methods are now available to distinguish and characterize members of MBTC species from the NTM. The SD Bioline TB AgMPT64 Rapid ${ }^{\circledR}$ is a sample and rapid immunochromatographic test used in differentiating Mycobacteria spp into Mycobacterium tuberculosis complex and non-tuberculosis mycobacteria (NTM) otherwise known as mycobacteria other than tuberculosis or environmental mycobacteria, while Genotype MBTC (Hain Life Science, Nehren, Germany) is a recently developed commercial DNA-strip assay used in differentiating MBTC isolated from cultured materials into their various species. The procedure involves isolating DNA from cultured material, multiplex amplification with biotinlyted primers and reverse hybridization of the single-stranded, biotin-labelled amplicons to membrane bound probes. The resulting banding patterns indicate the species of the isolated mycobacterium.

\section{Material and Methods}

Ethical clearance was written and obtained from the relevant authority for permission to carry out the work. The various hospitals were the samples were collected were informed officially via the ethical approval.

\section{Study Area}

Gombe state is located between latitude $9^{\circ} 30^{\prime}$ and $12^{\circ}$ $30^{\prime} \mathrm{N}$ and longitudes $8^{\circ} 45^{\prime}$ and $11^{\circ} 45^{\prime} \mathrm{E}$ of the Greenwich Meridian. It is on an altitude of $540 \mathrm{~m}$ above the sea level and covers an estimated land mass of $20,265 \mathrm{~km}^{2}$ and has a population of $1,820,415$ inhabitants. The State has two distinct climates, the dry season (December to March) and the rainy season (April to October) with an average rainfall of $850 \mathrm{~nm}$, with the mean maximum monthly temperature of $37^{\circ} \mathrm{C}$, relative humidity of $94 \%$ in August and $10 \%$ in December. Five General Hospitals were selected for this study. 1250 patients were included in this study and the WHO recommended kits called Determine, Unigold and Star pack was used based on the Manufacturer's instructions.

\section{Collection and culturing of sputum}

Three sputum samples were collected from patients who reported to the selected hospitals, on the spot and one each morning for two days. The sputum samples were then pooled together stored in Cetyl Pyrinidium Chloride (CPC) and data pertaining the patient such as age, sex and HIV status were obtained and recorded. All the sputum samples collected were transported to Zankli medical laboratory Abuja for culturing and analysis. Mycobacterial 


\section{Open Access Journal of Microbiology \& Biotechnology}

growth were monitored every week for up to 8 weeks and samples negative for AFB after 8 weeks were considered negative and discarded.

\section{SD Bioline TB AgMPT64 Analysis}

Positive acid fast bacilli on culture were subjected to SD Bioline analysis, according to the manufacturer's instructions. The test consists of test cassettes which consist of a sample pad, a gold conjugate, nitrocellular membrane and an absorbent pad. Mouse monoclonal antiMPT64 was immobilized on the nitrocellulose membrane as the capture material (test line). The cassette has a letter $\mathrm{T}$ and $\mathrm{C}$ as test line and control on the surface of the case. Positive result produced red to purple band.

\section{Genotype MBTC (Line probe assay)}

All samples that were positive on the SD Bioline that were MBTC species were then subjected to Genotype MBTC (line probe assay) which differentiated them into their various species. The procedures involved were carried out as instructed by the manufacturer. Amplification and hybridization controls verified the test procedures; an MTBC control confirmed the identification as MTBC. A template sheet showing the positions of the lines and the interpretation table, both provided with the kit, were used for interpretation of the test results

\section{Data Analysis}

Statistical package for social science (SPSS) package 20 versions was used to analyze the data.

\section{Results}

In this study, 100 sputum samples were collected and analyzed, $40(40 \%)$ were identified as Mycobacterium tuberculosis complex (MBTC) (Table1), of the 40 MBTC isolates subjected to genotype assay, 30(75\%) were $M$. $t b$, $6(15 \%)$ were $M$. bovis while $3(7.5 \%)$ were $M$. africanum, $1(2.5 \%)$ was unidentified using the standard banding patterns (Table 2). Hospital records of 1250 patients who reported to the hospitals during the period of the study were also analyzed, which showed that, age group between less than 40 years and males had the highest prevalence of $\mathrm{Tb}$ infection with $687(54.96 \%)$ than female with 563(45.04\%) there was statistical association between sex and also between age growth (Table 2). Based on the occupational activities of the patients, civil servant with $80(35.6 \%)$ had the highest TB prevalence followed by businessmen with the prevalence of $45(24.3 \%)$ and least was found among the student with the prevalence of 58(4.6\%). Similarly civil servants with 65(28.9\%) have the highest prevalence of HIV followed by the farmers with the prevalence of $42(19.0 \%)$ and the HIV seropositive was found among the commercial sex workers with the prevalence of $30(15.0 \%)$.

Co-infection of HIV/TB based on the occupation shows that commercial sex workers with $48(25.3 \%)$ had the highest HIV/TB co-infection followed by housewife with $15(8.5 \%)$ and the least co-infection of HIV/TB was found among the Business people with the prevalence of $9(4.9 \%)$ and this was shown on Table 3.

\begin{tabular}{|c|c|c|c|c|}
\hline & \multicolumn{2}{|c|}{ MBTC } & \multicolumn{2}{c|}{ NTM } \\
\hline Ag (Years) & Male & Female & Male & Female \\
\hline$>15$ & 0 & 0 & 0 & 0 \\
\hline $14-24$ & 3 & 1 & 2 & 3 \\
\hline $25-34$ & 15 & 3 & 12 & 6 \\
\hline $35-44$ & 9 & 2 & 14 & 5 \\
\hline $45-54$ & 4 & 1 & 4 & 2 \\
\hline $55-64$ & 2 & 0 & 0 & 0 \\
\hline$>65$ & 0 & 0 & $\mathbf{3 7}$ & $\mathbf{2 3}$ \\
\hline
\end{tabular}

Table 1: Age and sex distribution of cases with Mycobacterium tuberculosis complex and non tuberculous Mycobacterium 


\section{Open Access Journal of Microbiology \& Biotechnology}

\begin{tabular}{|c|c|c|c|c|}
\hline Location & Total for AFB+ & M. $\boldsymbol{t b}(\%)$ & M. bovis(\%) & M.africanum(\%) \\
\hline Zambuk & 15 & $18(45)$ & $3(7.5)$ & $2(5.0)$ \\
\hline Kaltungo & 5 & $2(5.0)$ & $0(0.0)$ & $0(0.0)$ \\
\hline Billiri & 5 & $3(7.5)$ & $1(2.5)$ & $1(2.5)$ \\
\hline Bajoga & 5 & $2(5.0)$ & $2(5.0)$ & $0(0.0)$ \\
\hline FMC Gombe & 10 & $5(12.5)$ & $0(0.0)$ & $0(0.0)$ \\
\hline Total & $\mathbf{4 0}$ & $\mathbf{3 0}$ & $\mathbf{6}$ & $\mathbf{3}$ \\
\hline
\end{tabular}

Table 2: Culture and Differentiation of Isolates.

\begin{tabular}{|c|c|c|c|c|}
\hline Location & Total No screened & TB + (\%) & HIV + (\%) & Co-infection (\%) \\
\hline Civil servant & 225 & $80(35.6)$ & $65(28.9)$ & $15(6.7)$ \\
\hline Housewife & 175 & $35(20.0)$ & $22(12.5)$ & $15(8.5)$ \\
\hline Student & 245 & $58(4.6)$ & $30(2.4)$ & $21(8.5)$ \\
\hline Farmer & 220 & $65(5.2)$ & $42(19.0)$ & $15(6.8)$ \\
\hline Business & 185 & $45(24.3)$ & $18(9.7)$ & $9(4.9)$ \\
\hline Commercial sex worker & 200 & $25(12.5)$ & $30(15.0)$ & $25(12.5)$ \\
\hline Total & $\mathbf{1 2 5 0}$ & & & \\
\hline
\end{tabular}

Table 3: Occupation of patients with co-infection of TB/HIV.

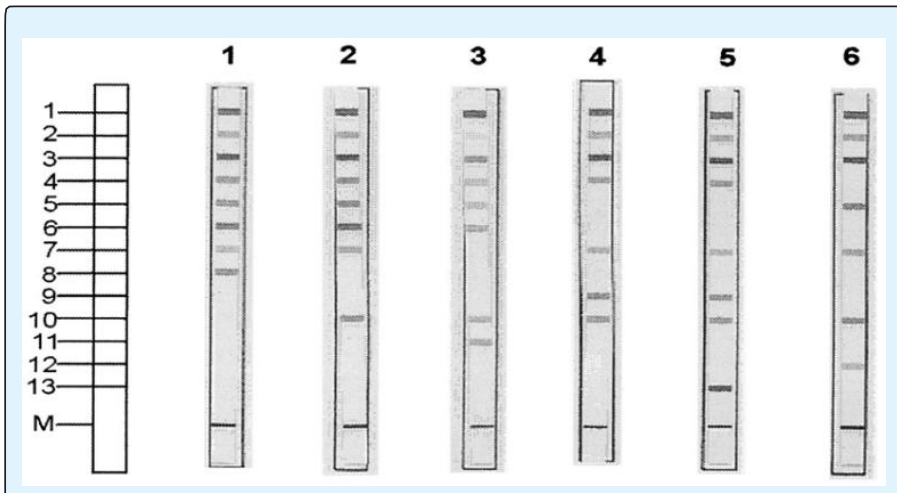

Figure 1: Results representative of all of the patterns obtained with the Genotype MTBC assay. The positions of the oligonucleotides and the marker line are shown on the left. The specificity and targetedgenes of the lines are as follows: 1, conjugate control; 2, plification control (23S rRNA); 3, MTBC specific (23S rRNA); 4 to 12, discriminative for the MTBC species (gyrB); 13, M. bovis BCG (RD1); M, marker line for correct orientation of the strip. Patterns of the strips shown: 1, M. tuberculosis or $M$. africanum subtype II or $M$. canetti. 2, M. africanum subtype I; 3, M. microti; 4, M. bovis subsp. bovis; $5, M$. bovisBCG; 6, M. bovis subsp. Caprae (Adapted from Richter et al., 2003)

\section{Discussion}

In this study, males were more infected with tuberculosis than females. Statistically, there was no significant difference in the distribution of tuberculosis and gender. This agrees with previous work by Alfred and Silas (2005) and Kehinde and Okesola (2010) [1,2] in the Southeast and Ibadan, Nigeria. Males are more exposed to source of infection than females hence the higher prevalence in males. Christopher et al. (2012) [3] observed that age group between 30-39 years are more affected which concur with the findings of this study. The present investigation has revealed the prevalence of tuberculosis in HIV seropositive patients in the selected General Hospitals in the study area. Percentage prevalence found in this study was higher than that reported by Olaniran et al. (2011) [4] in Obafemi Awolowo University Teaching Hospital Complex, Ile-Ife, Nigeria. These differences may be because the investigation was done on only HIV patients, while this study focused on new patients [5]. Age distribution of patients with co-infection of HIV and TB in this study revealed that age group 30-40yrs had the highest incidence. This concur with the report of Kamenju and Aboud (2011) [6] in Dares Salaam, Tanzania, and 
connected sexually active age group patients in which both TB and HIV prevail most. The other possible reason for this may be their increased family, organizational, and societal responsibilities as people in this age group involve themselves in various extraneous daily activities in order to win the socio-economic hardship that increases the frequency of their contact with other patients in their society. The present study reveals a high prevalence of Non-tuberculous Mycobacteria (NTM) otherwise known as environmental Mycobacterium in humans depicting the role of environment. Some patients in this study were livestock keepers especially cattle, and the cattle could have acquired the infection through contaminated environment during grazing or at water sources. Prevalence of NTM in this study is lower than previous report of $23.08 \%$ in Jos by Mwak et al. (2006) and Gumel et al. (2015) [7] in HIV/AIDS patients in Zaria, Nigeria. Mdegella et al. (2004) and Durnez et al. (2009) [8] reported prevalence of $14 \%$ and $19 \%$ respectively in milk samples. Although this study did not investigate milk and its products, a common practice in the study area is consumption of unpasteurized milk that might expose consumers to risk of infection. It is presumed the NTMs observed in this study might have predisposed some individuals to HIV/AIDS infection.

From this study, the majority of MTBC cases were caused by $M$. tuberculosis, $M$. bovis and $M$. africanum in the sputum samples analyzed. The proportion of $M$. africanum is low compared to previous reported studies done in some parts of Nigeria. For instance, Cadmus et al. (2006) [9] reported $13 \%$ in Southern Nigeria, while, Waziri et al. (2015) reported $10.8 \%$ in pulmonary patients in Zaria, Kaduna State. The reason for the low prevalence of $M$ africanum in this study could is due to the methodology. This study have shown that the combination of the two methods used can effective in the study area thereby reducing the time and cost of diagnosis in our hospitals.

\section{Conclusion}

The results of this study have highlighted the fact that TB and HIV infections are common in State, More studies, are required to isolate and differentiate MTBC from NTM using the clinical specimens for testing. The differentiation of the agents will go a long way in helping the clinicians in choice of drugs and course of treatments.

\section{References}

1. Itah AY, Udofia SM (2005) Epidemiology and Endemicity of Pulmonary Tuberculosis (PTB) in Southeastern Nigeria. Southeast Asian J Trop Med Public Health 36(2): 317-323.
2. Kehinde AO, Okesola AO (2010) Epidemiology of Clinical Isolates of Mycobacterium Tuberculosis at Ibadan, Nigeria. Nigerian Journal Physiology of Science 25(2): 135-138.

3. Christopher CA, Emeka k, Vivien OA (2012) The Pattern of Presentation and Prevalence of Tuberculosis in HIV-Seropositive Patients Seen at Benin City, Nigeria. International Scholarly Research Network Pulmonology: 6.

4. Olaniran O, Hassan-Olajokun RE, Oyovwevotu MA, Agunlejika RA (2011) Prevalence of Tuberculosis Among HIV/AIDS Patients in Obafemi Awolowo University Teaching Hospital International Journal of Current Microbiology of Applied Sciences 3(6): 831838.

5. Kassu A, Mengistu G, Ayele B, Diro E, Mekonnen F, et al. (2007) Coinfection and Clinical Manifestations of Tuberculosis in Human Immunodeficiency VirusInfected and Uninfected Adults at a Teaching Hospital. Northwest Ethiopian Journal of Microbiology and Immunology Infection 40: 116-122.

6. Kamenju P, Aboud S (2011) Tuberculosis-HIV CoInfection among Patients admitted at Muhimbili National Hospital in Dares Salaam, Tanzania. Tanzania Journal of Health Research 13(1): 21-26.

7. Mwak J, Gomwalk N, Bello C, Kandakai-Olukemi Y (2006) Human Pulmonary Infections with Bovine and Environment (Atypical) Mycobacteria in Jos, Nigeria. Ghana Medical Journal 40: 132-136.

8. Durnez L, Sadiki H, Katakweba A, Machang'u R, Kazwala R, et al. (2009) The Prevalence of Mycobacterium Bovis-Infection and Atypical Mycobacterioses in Cattle in and Around Morogoro, Tanzania. Tropical Animal Health and Production 41(8): 1653-1659.

9. Cadmus S, Palmer S, Melissa O, James D, Karen G, et al. (2006) Molecular Analysis of Human and Bovine Tubercle Bacilli from a Local Setting in Nigeria. Journal of Clinical Microbiology 44: 29-34.

10. Berhe KK, Demissie A, Kahsay AB, Gebru HB (2012) Diabetes Self-Care Practices and Associated Factors Among Type 2 Diabetic Patients in Tikuranbessa Specialized Hospital, Addis Ababa, Ethiopia- a Cross Sectional Study. IJPSR 3(11): 4219-4229. 


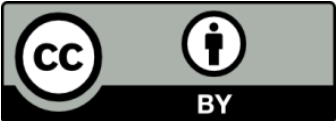

